•综述・

\title{
基于站点的生物多样性星空地一体化遥感监测
}

\author{
李爱农 ${ }^{1^{*}}$ 尹高飞 张正健 $^{1,2}$ 谭剑波 ${ }^{1,2}$ 南 希 ${ }^{1}$ 马克平 ${ }^{3}$ 郭庆华 $^{3}$
}

1 (中国科学院成都山地灾害与环境研究所, 数字山地与遥感应用研究中心, 成都 610041)

2 (中国科学院大学, 北京 100049)

3 (中国科学院植物研究所植被与环境变化国家重点实验室, 北京 100093)

摘要：科学制定生物多样性保护和恢复政策，需要空间上连续、时间上高频的物种和生境分布以及物种迁移信息 支持, 遥感是目前能满足该要求的有效技术手段。近年来, 遥感平台和载荷技术高速发展, 综合多平台、多尺度、 多模式遥感技术, 开展基于站点的星空地一体化遥感观测试验, 可以对地表进行时空多维度、立体连续观测, 为生 物多样性遥感监测提供了新的契机。本文总结了使用遥感技术监测生物多样性的主要方法, 回顾了典型的星空地 一体化遥感观测试验。综述以往研究发现, 一方面, 现有遥感试验还缺少对生物多样性直接监测指标的观测, 另一 方面, 生物多样性遥感监测方法也缺少星空地多维立体观测平台的支撑, 函需加强两者的融合, 开展基于站点的 生物多样性星空地一体化遥感监测研究。以设于我国四川王朗大熊猫国家级自然保护区内的王朗山地生态遥感综 合观测试验站为例, 展示了星空地一体化遥感综合观测试验平台在生物多样性监测中的应用潜力。星空地一体化 遥感观测可以提供物种和生境的综合定量信息, 与生态模型有机结合, 可以刻画生物多样性的时空格局与动态过 程, 有助于挖掘过程机理, 提高生物多样性监测的信息化水平。

关键词: 生物多样性; 星空地一体化观测; 遥感观测; 王朗

\section{Space-air-field integrated biodiversity monitoring based on experimental station}

\begin{abstract}
Ainong $\mathrm{Li}^{1 *}$, Gaofei Yin ${ }^{1}$, Zhengjian Zhang ${ }^{1,2}$, Jianbo $\operatorname{Tan}^{1,2}, \mathrm{Xi} \mathrm{Nan}^{1}$, Keping $\mathrm{Ma}^{3}$, Qinghua Guo ${ }^{3}$
1 Research Center for Digital Mountain and Remote Sensing Application, Institute of Mountain Hazards and Environment, Chinese Academy of Sciences, Chengdu 610041

2 University of Chinese Academy of Sciences, Beijing 100049

3 State Key Laboratory of Vegetation and Environmental Change, Institute of Botany, Chinese Academy of Sciences, Beijing 100093
\end{abstract}

\begin{abstract}
Developing effective policies for biodiversity conservation and restoration policies requires spatially and temporally explicit data on distribution of species and habitats. Remote sensing provides an effective technical tool to meet this requirement. In recent years, the rapid development of integrated multi-platform, multi-scale, multi-mode remote sensing technology the implementation of integrated remote sensing observations across space-air-field provides novel opportunities for biodiversity monitoring. In this paper, we review the main methods of remote sensing that aids biodiversity monitoring and assess existing remote sensing observation experiments. We found that current methods of biodiversity monitoring using remotely-sensed data lacked the support of space-air-field integrated observations and the existing space-air-field integrated observations did not include biodiversity parameters. The Wanglang integrated observation and experiment station for mountain ecological remote sensing illustrates the potential to integrate experimental station-based and space-air-field integrated observations for biodiversity monitoring. Our review highlights that integrating direct observations with remote sensing can provide spatio-temporally explicit information on species and habitats and improve the informed monitoring of biodiversity.
\end{abstract}

Key words: biodiversity; space-air-field integrated observation; remote sensing; Wanglang 
生物多样性是生物及其与环境形成的生态复 合体以及与此相关的各种生态过程的总和(蒋志刚 和马克平, 2009)。作为地球上重要的资源宝库, 生 物多样性直接为人类及相关生物提供各种需求, 并 通过发挥其相关的功能, 间接地为地球生物提供适 宜的栖息环境以及各种服务(Cardinale et al, 2012)。 由于全球人口大幅度增加, 人类向自然索取的物质 和服务过多, 加之全球气候变化的影响, 导致全球 范围内的生物多样性水平大幅下降(马克平和钱迎 倩, 1998; Pimm et al, 2014)。生物多样性的大量丧失 进一步影响到人类福祉和生态系统健康, 已成为当 今重要的全球性问题(Ceballos et al, 2015; 马克平, 2016)。

生物多样性监测是实施生物多样性保护行动 计划和开展生物多样性修复的基础。原位定点观测 可以提供生态系统、群落、物种、基因等多尺度的 生物多样性信息, 是生物多样性监测中不可或缺的 技术手段。但是该类基于实地调查的方法难以获得 大空间覆盖、长时间序列的生物多样性信息, 且受 制于研究区通达性、人力物力成本等多重限制。近 年来, 遥感平台和载荷技术高速发展, 星基、空基、 地基等多平台、多尺度、多模式遥感技术层出不穷, 可以对地表要素和过程进行时空多维度、立体观 测。遥感已经成为广受认可的生物多样性监测方法 (胡海德等, 2012)。然而已有的生物多样性遥感监测 方法大都基于单一平台, 缺乏对星空地多维立体遥 感平台的集成应用，限制了其应用潜力。

星空地一体化遥感综合观测以地面试验站为 依托，协同原位定点观测和多维立体遥感平台，可 以收集生物多样性-遥感观测配对数据, 支撑由生 物多样性到遥感观测的前向模拟和由遥感观测到 生物多样性的数学推断, 有望进一步提高生物多样 性监测水平。然而, 已有星空地一体化遥感观测试 验缺少生物多样性的监测指标, 亟需加强生物多样 性遥感监测方法和星空地一体化遥感观测试验之 间的有机融合。本文在此背景下综述两者研究进展, 并分析两者结合的可能途径, 希望进一步提高生物 多样性遥感监测水平。

\section{生物多样性遥感监测方法研究进}

当前, 基于遥感技术的生物多样性监测方法大 体可以分为直接和间接两种途径(Turner et al,
2003)。直接法是根据生物多样性的遥感诊断特征直 接识别物种或群落类型及其分布; 间接法是通过遥 感数据衍生的与生物多样性密切相关的指标或变 量, 结合野外采样, 构建模型来预测物种分布以及 多样性格局。生物多样性遥感监测的直接法和间接 法主要平台和监测内容见表 1 。

\section{1 直接法}

直接法通过遥感数据直接识别物种的类型和 数目, 从而获取区域物种的丰度和多度。由于各个 物种在光谱上有一定的差别, 因此可以依据光谱特 征判断出不同的类型，直接识别物种和物种分布 (魏彦昌等, 2008)。此外, 纹理信息也可以用于物种 识别, 实际应用时一般需要结合高光谱和高空间分 辨率遥感数据才能识别出物种的类型(胡海德等, 2012)。

近年来, 随着传感器和无线通信技术的发展, 出现了多种基于地基遥感的生物多样性监测方法, 如红外相机(肖治术等, 2014)、视频监控(Burton et al, 2015)和声景监测(Towsey et al, 2014)等。该方法通 过在生物多样性丰富的区域布设照相机、摄像机和 录音机等方式，通过拍照和录音直接获取物种的状 况和空间分布信息，是一种对野生动物进行非损伤 性采样的技术。该方法已成为研究动物多样性的常 用手段(张履冰等, 2014)。

\section{2 间接法}

间接法是目前生物多样性遥感监测的主流方 式, 主要包括基于景观指数(Myers et al, 2000)、遥 指数(Petrou et al, 2015)、光谱变异性指数(Herrmann et al, 2011)以及基于模型模拟(Nagendra, 2001)的生 物多样性监测等(表1)。

利用土地覆盖分类信息得到景观指数开展生 物多样性制图在实践中得到了较多的应用。Forman 和Gordon (1986)认为各种景观指数与木本植物物 种丰富度具有相关性, 之后的很多研究都支持中尺 度物种丰富度与景观异质性相关的观点 (Gould \& Walker, 1997)。另一方面, 许多研究也表明, 生态系 统的面积与其栖息的物种数目密切相关, 生态系统 面积等景观指数的变化直接影响生态系统维持物 种多样性的能力。IUCN生态系统红色名录等评估 体系常将遥感获取的景观指数作为生态系统水平 上生物多样性监测的重要指标(Keith et al, 2013; 朱 超等, 2015)。因此，应用遥感方法对这二者的相关 
表1 生物多样性遥感监测的主要方法

Table 1 Main methods of remote sensing for biodiversity monitoring

\begin{tabular}{|c|c|c|c|c|c|}
\hline $\begin{array}{l}\text { 方法 } \\
\text { Method }\end{array}$ & $\begin{array}{l}\text { 传感器 } \\
\text { Sensor }\end{array}$ & $\begin{array}{l}\text { 空间分辨率 } \\
\text { Spatial resolution }\end{array}$ & $\begin{array}{l}\text { 光谱分辨 } \\
\text { Spectral resolution }\end{array}$ & $\begin{array}{l}\text { 监测内容 } \\
\text { Monitoring contents }\end{array}$ & $\begin{array}{l}\text { 参考文献 } \\
\text { Reference }\end{array}$ \\
\hline \multicolumn{6}{|c|}{ 直接法 Direct approaches } \\
\hline \multirow[t]{2}{*}{$\begin{array}{l}\text { 天基遥感 } \\
\text { Space-based }\end{array}$} & $\begin{array}{l}\text { IKONOS } \\
\text { Quickbird } \\
\text { WorldView-2 } \\
\text { 吉林一号 Jilin-1 }\end{array}$ & $\begin{array}{l}1-4 \mathrm{~m} \\
0.6-2.4 \mathrm{~m} \\
0.5 \mathrm{~m}, 2 \mathrm{~m} \\
0.72-2.88 \mathrm{~m}\end{array}$ & $\begin{array}{l}\text { 4波段 } 4 \text { bands } \\
\text { 4波段 } 4 \text { bands } \\
\text { 8波段 } 8 \text { bands } \\
\text { 4波段 } 4 \text { bands }\end{array}$ & $\begin{array}{l}\text { 冠层、物种信息 } \\
\text { Canopy and } \\
\text { species information }\end{array}$ & $\begin{array}{l}\text { Bejarano et al, } 2010 \\
\text { Wulder et al, } 2008 \\
\text { Petrou et al, } 2014\end{array}$ \\
\hline & $\begin{array}{l}\text { ADS } 40 \\
\text { AHS-160 } \\
\text { CASI } \\
\text { Lidar }\end{array}$ & $\begin{array}{l}0.2 \mathrm{~m} \\
2.4 \mathrm{~m} \\
1 \mathrm{~m}\end{array}$ & $\begin{array}{l}\text { 4波段 } 4 \text { bands } \\
\text { 63波段 } 63 \text { bands } \\
\text { 288波段 } 288 \text { bands }\end{array}$ & & $\begin{array}{l}\text { Forzieri et al, } 2013 \\
\text { Delalieux et al, } 2012 \\
\text { Belluco et al, } 2006 \\
\text { Guo et al, } 2016\end{array}$ \\
\hline $\begin{array}{l}\text { 地基遥感 } \\
\text { Ground-based }\end{array}$ & $\begin{array}{l}\text { 红外相机 Infrared camera } \\
\text { 视频监控 Video monitoring } \\
\text { 声景监控 Soundscape }\end{array}$ & & $\begin{array}{l}\text { 可见光、红外 } \\
\text { Visible, infrared } \\
\text { 可见光、红外 } \\
\text { Visible and infrared }\end{array}$ & $\begin{array}{l}\text { 物种活动场景 } \\
\text { The behavior of species }\end{array}$ & $\begin{array}{l}\text { Xiao et al, } 2014 \\
\text { Burton et al, } 2015 \\
\text { Towsey et al, } 2014\end{array}$ \\
\hline \multicolumn{6}{|c|}{ 间接法 Indirect approaches } \\
\hline $\begin{array}{l}\text { 景观指数法 } \\
\text { Landscape index }\end{array}$ & $\begin{array}{l}\text { TM/ETM + } \\
\text { HJ } \\
\text { MODIS }\end{array}$ & $\begin{array}{l}30-120 \mathrm{~m} \\
30-100 \mathrm{~m} \\
250-1 \mathrm{~km}\end{array}$ & $\begin{array}{l}\text { 7-9波段 } 7-9 \text { bands } \\
\text { 4波段 } 4 \text { bands } \\
\text { 36波段 } 36 \text { bands }\end{array}$ & $\begin{array}{l}\text { 土地分类 } \\
\text { Land cover classification }\end{array}$ & $\begin{array}{l}\text { Zhong et al, } 2014 \\
\text { Wang et al, } 2012 \\
\text { Fang et al, } 2011\end{array}$ \\
\hline $\begin{array}{l}\text { 遥感指数法 } \\
\text { Satellite index }\end{array}$ & $\begin{array}{l}\text { EO-1 Hyperion } \\
\text { ASTER } \\
\text { Sentinel-2 MSI }\end{array}$ & $\begin{array}{l}30 \mathrm{~m} \\
15 \mathrm{~m}, 30 \mathrm{~m}, 90 \mathrm{~m} \\
10 \mathrm{~m}, 20 \mathrm{~m}, 60 \mathrm{~m}\end{array}$ & $\begin{array}{l}220 \text { 波段 } 220 \text { bands } \\
14 \text { 波段 } 14 \text { bands }\end{array}$ & $\begin{array}{l}\text { 多样性指数 } \\
\text { Biodiversity index }\end{array}$ & $\begin{array}{l}\text { Pu et al, } 2010 \\
\text { Reiche et al, } 2012\end{array}$ \\
\hline $\begin{array}{l}\text { 光谱变异性指数法 } \\
\text { Spectral } \\
\text { heterogeneity index }\end{array}$ & $\begin{array}{l}\text { SPOT } \\
\text { CBERS-04 }\end{array}$ & $\begin{array}{l}1.5 \mathrm{~m}, 6 \mathrm{~m}, 1 \mathrm{~km} \\
5-80 \mathrm{~m}\end{array}$ & $\begin{array}{l}\text { 13波段 } 13 \text { bands } \\
\text { 4-6波段 } 4-6 \text { bands } \\
\text { 12波段 } 12 \text { bands }\end{array}$ & $\begin{array}{l}\text { 光谱异质性指数 } \\
\text { Spectral heterogeneity } \\
\text { index }\end{array}$ & $\begin{array}{l}\text { Herrmann et al, } 2011 \\
\text { Guyon et al, } 2011\end{array}$ \\
\hline $\begin{array}{l}\text { 模型模拟法 } \\
\text { Model simulation }\end{array}$ & $\begin{array}{l}\text { TRMM/TMI } \\
\text { FY-3C/VIRR }\end{array}$ & $\begin{array}{l}5-72 \mathrm{~km} \\
1 \mathrm{~km}\end{array}$ & $\begin{array}{l}5 \text { 波段 } 5 \text { bands } \\
10 \text { 波段 } 10 \text { bands }\end{array}$ & $\begin{array}{l}\text { 物种分布 } \\
\text { Species distribution }\end{array}$ & Zainuddin et al, 2006 \\
\hline
\end{tabular}

性进行测定，在生物多样性评价与保护方面可以发 挥重要的价值。这种方法适合较大的空间尺度, 单 纯使用这种方法得到的多样性信息精度不高 (Gillespie, 2005)。

基于遥感指数的生物多样性监测主要包括通 过生物量、归一化植被指数(normalized difference vegetation index, NDVI)等能指示生物多样性水平 的遥感指数及其时间序列变化来表征区域生物多 样性水平。许多研究表明, 生物量和NDVI等遥感指 数与生物多样性之间存在显著正相关性, 即生产力 假说(Productivity Hypothesis) (Oindo \& Skidmore, 2002; Hooper et al, 2005)。然而, 也有研究表明此类 方法不完全适用, 例如Rocchini和Chiarucci (2004) 发现, NDVI与物种丰富度程度的关系很小。虽然生 产力与生物多样性的关系需进一步探讨, 但已有大 量的研究使用遥感指数监测物种多样性取得了较 好的效果。随着激光雷达(light detection and ranging, Lidar)等能获取地物精细空间结构的遥感技术的兴 起, 与生物多样性相关的遥感指数也越来越多, 该
方法将扮演更重要的角色(郭庆华等, 2016)。

使用光谱异质性指数监测生物多样性，是基于 光谱变异假说 (spectral variation hypothesis, SVH) (Palmer et al, 2002), 即使用光谱异质性表征栖息地 的景观复杂性，而高度复杂的栖息地可容纳更多的 物种。光谱异质性假说的量化以及遥感波段的选择 是该方法需要解决的重要问题(Carlson et al, 2007; Rocchini, 2007)。

基于模型模拟的生物多样性监测主要以遥感 数据、环境数据和干扰为基础，通过相关的算法和 模型，模拟物种的空间分布(魏彦昌等, 2008)。目前 比较成熟的模拟模型有最大熵值算法、遗传算法和 神经网络等(Foody \& Cutler, 2006; Saatchi et al, 2008)。该类方法可以获取物种的潜在分布区域，广 泛应用于就地和迁地保护研究中(Phillips \& Dudík, 2008; Soberon \& Nakamura, 2009)。然而，该方法仅 能模拟某一物种潜在的空间分布概率，与实际的空 间分布仍然有一定差异; 同时, 模拟结果仅能反映 某一物种生物多样性的多度。 


\section{星空地一体化遥感综合观测研}

星空地一体化遥感综合观测是指以地面试验 站为依托, 协同星基、空基和地基观测设备, 对研 究区开展生态、水文、社会经济等多重属性的综合 观测。它可以提供研究区的多空间尺度、长时间跨 度的立体观测, 从而为局地生态机理的升尺度、大 尺度情况下过程模型的参数化等提供支撑, 也是探 索遥感与生物多样性信息的机理关联, 进一步提高 生物多样性监测水平的潜在技术手段。近年来, 随 着对地理过程高时空分辨率监测需求的提升, 以及 各种新型观测技术和手段的不断涌现, 星空地一体 化遥感综合观测有力支撑了遥感科学及地学应用。

\section{1 支撑地球表层系统研究}

地球表层系统将地球各大圈层视为自然和社 会的综合体, 是人类圈与地球相互作用的复合系 统。星空地一体化遥感观测所提供的多维立体观测, 为地球表层系统研究提供了有力支撑。

泰加林生态系统-大气研究计划(Boreal Ecosystem-Atmosphere Study, BOREAS)是一项典型的 面向地球表层系统研究的一体化试验, 它是由美国 国家宇航局主导的大型国际研究计划(Sellers et al, 1995), 主要试验目标包括: (1)增强泰加林-大气边 界层之间辐射、水热、碳、痕量气体等过程模拟能 力。(2)基于遥感与其他集成建模技术, 发展大尺度 过程模拟模型。BOREAS试验主要集中在1994-1996 年, 收集了大量地基、塔基、空基和星基观测数据, 并取得了诸多研究成果(Sellers et al, 1997)。虽然 BOREAS计划历史久远, 但其发展了许多超前的观 测理念, 如多尺度嵌套观测方式, 在后续星空地一 体化遥感观测试验中得到了广泛应用。

\section{2 支撑流域科学研究}

流域是开展地球系统科学实践的一个单元, 流 域科学是流域尺度上的地球系统科学, 目标是理解 和预测流域复杂系统的特征和过程, 服务流域可持 续发展。建立精细、实时、星空地一体化的流域观 测系统可用于探寻流域复杂系统多尺度特征和运 行规律(程国栋和李新, 2015)。

以黑河遥感试验研究站为依托, 我国先后开展 了“黑河综合遥感联合试验”(Watershed Allied Telemetry Experimental Research, WATER)(Li et al,
2009)和“黑河流域生态-水文过程综合遥感观测联 合试验”(Heihe Watershed Allied Telemetry Experimental Research, HiWATER)(Li et al, 2013)。试验的 总体目标是显著提升对流域生态和水文过程的观 测能力, 建立国际领先的流域观测系统, 提高遥感 在流域生态-水文集成研究和水资源管理中的应用 能力。WATER和HiWATER计划获取了大量多尺度 地面观测和遥感反演生态水文参量数据, 在尺度转 换、产品生产与真实性检验等多个方面，极大地促 进了我国定量遥感基本理论与方法的发展(晋锐等, 2017), 也为丰富流域科学的方法论做出了贡献(程 国栋和李新, 2015)。

\section{3 支撑多尺度多波段遥感机理研究}

多模式遥感观测的集成应用可以提供陆表多 维互补信息，丰富陆表参数反演的类型，提高反演 精度。载荷平台和传感器的发展, 为多模式遥感的 集成应用提供了技术保证，但多模式遥感集成还缺 乏必要的理论基础。开展星空地一体化观测支持下 的多模式遥感观测试验，可为全波段遥感机理模型 提供标定和验证所需的数据(Zhang et al, 2017)。

怀来遥感综合试验站位于河北省怀来县, 2004 年建成投入使用, 隶属于中国科学院特殊环境与灾 害监测网络。2014年，在怀来站支撑下开展了全波 段多尺度遥感机理综合试验。该试验由遥感科学国 家重点试验室组织实施，科学目标为：获取全波段 多尺度遥感辐射、散射特性测量与配套数据集, 验 证全波段遥感辐射与散射模型，为全波段多尺度遥 感模拟系统的研发提供真实场景和数据支撑。该试 验具体实施了星空地一体化的协同观测，对一体化 观测的组织实施也具有借鉴意义。

\section{4 支撑遥感产品真实性检验}

真实性检验是遥感产品应用及算法升级的前 提，而地面实测数据又是真实性检验的前提。地面 测量与遥感产品之间的观测足迹差异明显, 加之地 表空间异质性的影响，遥感产品的真实性检验需要 克服尺度差异引起的不确定性。星空地一体化综合 观测可以提供多尺度观测数据, 可支撑尺度转换模 型构建与验证, 从而得到遥感像元尺度真值, 检验 遥感产品质量。

“大脚印” (BigFoot)计划是一项典型的面向遥 感产品真实性检验的星空地一体化观测。它由美国 宇航局发起, 主要目标为集成地面观测、通量观测、 
遥感观测及生态过程模型, 验证中低分辨率的 MODIS陆表碳循环遥感产品(Cohen et al, 2006)。

BigFoot由 9 个观测站组成, 涵盖北美主要的 8 种植 被类型。每个Bigfoot站点面积均为 $5 \mathrm{~km} \times 5 \mathrm{~km}$, 且 都设有浴度相关高塔。土地覆被、叶面积指数等地 面离散点观测, 都通过Landsat数据转化为空间连续 的“真值”图。集成该“真值”图、通量观测数据, 以 及生态过程模型, 进一步可以得到各站点的 NPP “真值”。这些“真值”图可以作为遥感产品验证时的 参考标准(Cohen et al, 2006)。BigFoot在地面离散点 观测、通量足迹观测的升尺度(Wythers et al, 2003)、 地面观测与生态模型协同(Turner et al, 2005)等方面, 为星空地一体化遥感观测提供了许多可供参照的 规范和案例。

\section{3 已有研究存在的问题}

目前，遥感已广泛应用于生物多样性监测领域， 各种新方法和成功案例层出不穷(Bush et al, 2017)。 但文献调研发现，已有监测大都使用单一遥感观测 平台，缺乏对星空地多维立体遥感平台的集成应用， 限制了遥感在生物多样性监测中的应用潜力。例如, 在使用卫星遥感监测生物多样性时, 由于空间分辨 率不足, 不能给出精细的生物多样性信息, 并且生 物多样性信号与遥感信号之间的物理链接尚不清 晰; 使用近地遥感时, 可以提供精细的生物多样性 信息，但时间跨度和空间覆盖不足，难以提供时空 连续的生物多样性信息。开展生态系统水平上的生 物多样性评估时, 如何利用遥感数据确定生态系统 的非生物环境以及生物过程的退化风险(谭剑波等, 2017), 如何将评估结果应用到生物多样性保护实 践中, 仍需要进一步研究(Tan et al, 2017)。

随着遥感平台和载荷技术高速发展, 综合多平 台、多尺度、多模式遥感, 开展的星空地一体化遥 感观测试验, 有助于集成多平台优势 (胡天宇等, 2018)。已有星空地一体化观测试验, 在提升遥感机 理科学认知、地理过程监测水平方面发挥了重要作 用，同时也为生物多样性监测提供了一种潜在的技 术手段。但是, 现有站点大多关注遥感科学的本身, 以及基于遥感技术的碳水循环过程关键参量观测, 直接应用于生物多样性监测时还存在以下问题:

(1)已有星空地一体化观测试验的观测内容虽 然包含生物多样性相关的生境信息, 但观测设计的
初衷并非直接面向生物多样性遥感监测研究。借助 生产力假说或光谱异质性假说, 可以由星空地一体 化观测推断站点尺度的生物多样性。然而, 若要从 这些观测直接反映站点的生物多样性，还需要补充 物种丰度和多度等生物多样性的直接度量指标。而 且，上述现有观测站点，因为其根本目标不是关注 生物多样性本身, 其地理位置多数也不属于生物多 样性热点区域，难以借助现有观测来构建遥感信息 与生物多样性的机理关联。

(2)未考虑地形对遥感成像和生态过程的影响。 山区微气候特性复杂，生境优渥，孕育了种类繁多 的物种, 是生物多样性研究的的热点区域。但是, 山区交通状况恶劣, 不便于开展大范围的地面调查, 遥感技术在山区生物多样性调查中具有无可比拟 的潜力。若使用遥感技术开展山区生物多样性调查, 首先需要减少遥感影像中的地形效应，并考虑地形 对生态过程的影响。已有星空地一体化观测试验多 选择在地形平坦地区开展，化繁为简，较少关注复 杂山区重力梯度导致生态系统特有的物质循环过 程, 以及由此产生的山区特有遥感成像过程。因此, 建立山区星空地一体化综合观测试验站，研究遥感 成像和生态过程中的地形效应，探索构建遥感信号 与生物多样性之间的关联，对于提升山区生物多样 性监测水平至关重要。

综合星空地一体化的多维立体信息, 有助于提 高由生物多样性到遥感观测的前向机理认知, 获得 信息丰富、时空连续的生物多样性信息，从而发展 生物多样性遥感表征的时空尺度扩展模型, 实现生 物多样性遥感监测从站点尺度到区域尺度的时空 尺度扩展, 可能是当前进一步提高生物多样性遥感 监测水平可能的突破方向之一。

\section{实例分析：王朗山地生态遥感综合观测}

王朗山地生态遥感综合观测试验站(以下简称 王朗站)(Yin et al, 2017)位于四川王朗国家级自然保 护区(以下简称王朗保护区)内, 由中国科学院、水利 部成都山地灾害与环境研究所和王朗保护区管理 局共建，于 2017 年 6 月挂牌成立。王朗保护区 $\left(103^{\circ} 55^{\prime}-104^{\circ} 10^{\prime} \mathrm{E}, 32^{\circ} 49^{\prime}-33^{\circ} 02^{\prime} \mathrm{N}\right)$ 位于四川绵阳 平武县境内, 是我国较早建立的以保护大熊猫等珍 稀野生动物及其栖息地为主的国家级自然保护区 之一, 地处全球生物多样性核心地区的喜马拉雅一 
横断山区，保存了原始的自然生态系统和丰富的生 物多样性。王朗保护区有脊椎动物 22 目 64 科 200 种, 其中国家一级保护动物 11 种, 国家二级保护动物 23 种; 高等植物97科296属615种。保护区属丹巴一松潘 半湿润气候, 年均温 $1.5-2.9^{\circ} \mathrm{C}$, 年降水量 800-825 $m m$ 。海拔 2,400-4,980 m, 植被垂直带谱明显, 从低 到高依次分布着落叶阔叶林、针阔混交林、暗针叶 林、灌从草甸、草甸(王开运, 2004)。

王朗站的建设目标是: 构建具有国际影响力的 地-空-天立体化综合观测试验平台，提高山地复杂 环境下多源、多尺度、多平台地表观测数据的获取 能力, 促进山地定量遥感机理研究及其与山地生态 环境研究的深度融合, 丰富山地科学大数据平台建 设内涵, 支撑山地科学研究发展, 最终建设成为集 山地遥感理论、生态环境评估、植被生理生态、生 物多样性等为一体的综合性研究基地。

王朗站现阶段关注的核心科学问题包括山地 辐射传输建模、山地地表参数遥感反演与验证、生 物多样性遥感监测、山地生态系统对气候变化响应 与适应机制等。除此之外, 王朗站也是实践星空地 一体化遥感观测与生物多样性监测有机融合, 开展 基于站点的生物多样性星空地一体化遥感监测研
究的理想试验区, 可为大熊猫等珍贵物种保护提供 遥感科技支撑(图1)。在生物多样性监测方面，王朗 站关注以下科学问题:

(1)生物多样性监测中的假说检验。“光谱变异 假说”和“生产力假说”等假说是生物多样性监测的 间接法中的关键理论依据。依托王朗站，将开展系 统性的星空地多平台协同、植被生理生化-遥感多 光谱等多参数集成的观测试验。在森林动态大样方 内将收集物种多度、丰度等生物多样性信息，以及 配套的光谱信息、植被生产力信息。通过长时间序 列观测，可以支撑检验“光谱变异假说”、“生产力假 说”等在王朗试验站的合理性。

(2)基于LiDAR、成像光谱仪等的生境监测。采 用地基LiDAR收集的点云数据, 将重建森林动态大 样方三维真实场景, 实现样方内植被结构参数, 如 胸径、冠幅、叶面积指数、叶倾角分布等的高精度、 高分辨率估算。还将借助成像光谱仪等高光谱设备, 收集研究区内精细光谱数据, 发展基于光谱信息的 植被化学参数，如叶绿素含量、含水量等的遥感估 算模型。联合遥感获得的植被结构参数、生理生化 参数, 实现研究区生境的评估与动态监测。

(3)基于无人机的物种识别。物种自动识别是生

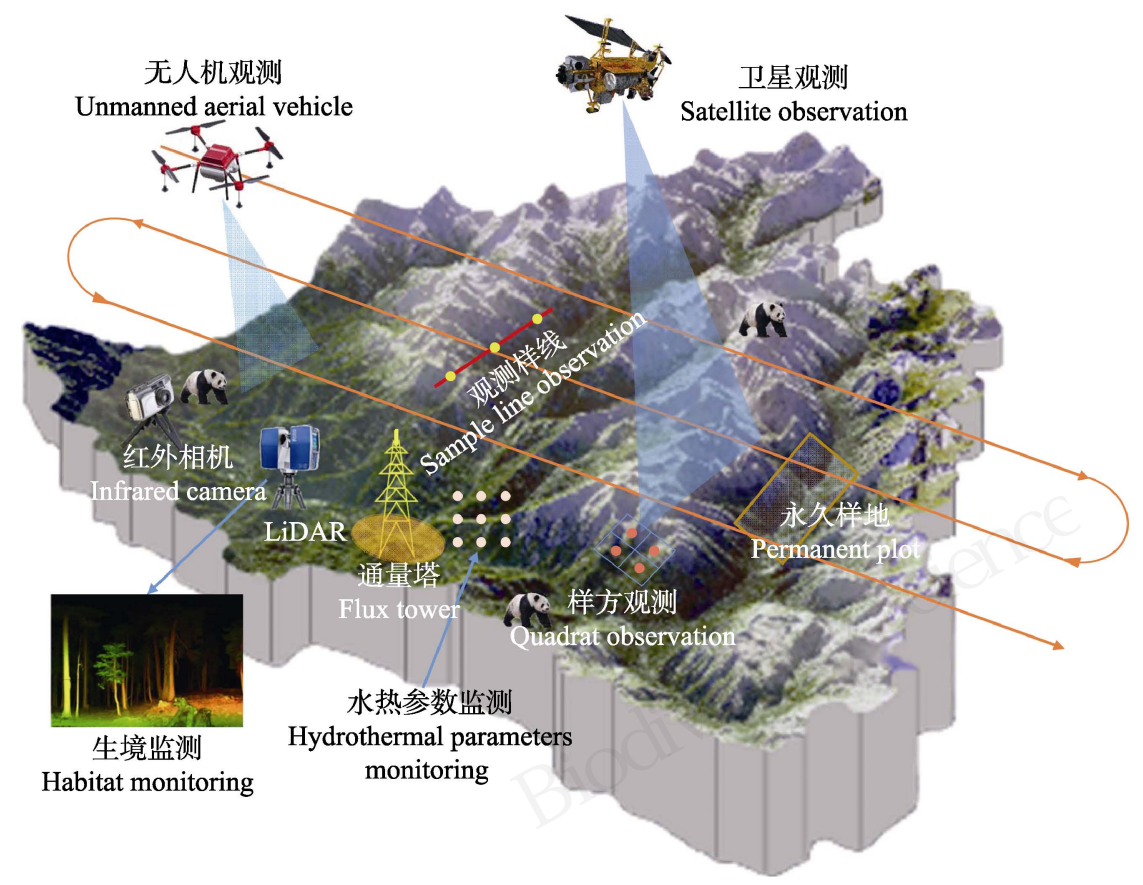

图1 基于站点的星空地一体化王朗山地生态遥感综合观测试验站生物多样性监测概念设计

Fig. 1 The concepted design of biodiversity monitoring at Wanglang integrated observation and experiment station based on space-air-field integrated remote sensing observation 
物多样性监测领域的难点。物种识别需要高空间分 辨率、高光谱分辨率影像的支持, 借以分辨各物种 特有的几何、纹理、光谱等诊断特征。使用低空无 人机, 搭载成像光谱仪, 可以获得高空间分辨率的 高光谱影像。结合地面调查, 构建各物种的识别特 征库, 可以辅助发展基于无人机遥感的物种自动识 别技术。

(4)人类活动对生境和珍稀物种的影响。王朗站 周围居民多以自由放牧为生, 偶有牲畜会进入保护 区内。近年来, 放牧对保护区内生境及大熊猫等珍 稀物种造成了不良影响。如Li等(2017)通过系统的 野外调查, 为家畜佩戴GPS卫星定位项圈, 利用保 护区长期监测数据, 结合模型模拟等技术手段, 分 析了牲畜影响下的大熊猫栖息地变化。研究发现, 过度放牧会威胁竹子更新, 加剧大熊猫栖息地的质 量退化和碎片化, 阻碍栖息地之间的连通性。王朗 山地生态遥感综合观测试验站为使用新信息技术 手段来研究人类活动对生境和珍稀物种的影响提 供了不可多得的理想试验区。

\section{5 结语}

本文总结了使用遥感技术监测生物多样性的 主要方法, 围绕星空地一体化遥感综合观测支持下 的研究案例, 回顾了典型的遥感观测试验, 并以王 朗山地生态遥感综合观测试验站为例, 论述了一体 化遥感综合观测试验在生物多样性监测中的应用 潜力。遥感是研究生物多样性的有效技术手段, 单 独使用单一遥感平台目前还存在较大的不确定性, 亟需开展基于站点的生物多样性星空地一体化遥 感监测试验。

已有星空地一体化观测试验大多关注遥感科 学自身基本理论的提升, 缺少生物多样性相关监测 指标。王朗山地生态系统遥感综合观测试验站是开 展面向生物多样性监测的星空地一体化观测试验 的有效尝试。面向生物多样性监测需求，对生物多 样性指标进行多平台集成、多尺度嵌套的立体观测, 收集生物多样性-遥感观测配对数据, 借助生态过 程模型、机器学习等手段, 建立由生物多样性到遥 感观测的前向机理模拟, 及由遥感观测到生物多样 性的数学推断, 可能是进一步提高生物多样性遥感 监测水平的潜在突破方向之一。

\section{参考文献}

Bejarano S, Mumby PJ, Sotheran I (2010) Predicting structural complexity of reefs andish abundance using acoustic $r$ emote sensing (RoxAnn). Marine Biology, 158, 489-504.

Belluco E, Camuffo M, Ferrari S, Modenese L, Silvestri S, Marani A, Marani M (2006) Mapping salt-marsh vegetation by multispectral and hyper-spectral remote sensing. Remote Sensing of Environment, 105, 54-67.

Burton A, Neilson E, Moreira D, Ladle A, Steenweg R, Fisher J, Bayne E, Boutin S, Stephens P (2015) Wildlife camera trapping: A review and recommendations for linking surveys to ecological processes. Journal of Applied Ecology, 52, 675-685.

Bush A, Sollmann R, Wilting A, Bohmann K, Cole B, Balzter H, Martius C, Zlinszky A (2017) Connecting earth observation to high-throughput biodiversity data. Nature Ecology \& Evolution, 1, 176.

Cardinale J, Duffy J, Gonzalez A, Hooper D, Perrings C, Venail P, Narwani A, Mace G, Tilman D, Wardle D (2012) Biodiversity loss and its impact on humanity. Nature, 486, 59-67.

Carlson K, Asner G, Hughes R, Ostertag RM (2007) Hyperspectral remote sensing of canopy biodiversity in Hawaiian lowland rainforests. Ecosystems, 10, 536-549.

Ceballos G, Ehrlich P, Barnosky A, García A, Pringle R, Palmer T (2015) Accelerated modern human-induced species losses: Entering the sixth mass extinction. Science Advances, 1, e1400253.

Cheng GD, Li X (2015) Integrated research methods in watershed science. Science China: Earth Sciences, 58, 1159-1168. (in Chinese with English abstract) [程国栋, 李 新 (2015) 流域科学及其集成研究方法. 中国科学: 地球 科学, 58, 1159-1168.]

Cohen WB, Maiersperger TK, Turner DP, Ritts WD, Pflugmacher D, Kennedy RE, Kirschbaum A, Running SW, Costa M, Gower ST (2006) MODIS land cover and LAI collection 4 product quality across nine sites in the western hemisphere. IEEE Transactions in Geosciences and Remote Sensing, 44, 1843-1857.

Delalieux S, Somers B, Haest B, Spanhove T, Borre V, Mucher C (2012) Heathland conservation status mapping through integration hyperspectral mixture analysis and decision tree classifiers. Remote Sensing of Environment, 126, 222-231.

Fang H, Liang S, Hoogenboom G (2011) Integration of MODIS LAI and vegetation index products CSM-CERES-Maize model for corn yield estimation. International Journal of Remote Sensing, 32, 1039-1065.

Foody GM, Cutler M (2006) Mapping the species richness and composition of tropical forests from remotely sensed data with neural networks. Ecological Modelling, 195, 37-42.

Forman R, Godron M (1986) Landscape Ecology. John Wiley \& Sons, New York.

Forzieri G, Tanteri L, Moser G, Catani F (2013) Mapping 
natural and urban environments using airborne multi-sensor ADS40-MIVIS-LiDAR synergies. International Journal of Applied Earth Observation and Geoinformation, 23, 313-323.

Gillespie T (2005) Predicting woody-plant species richness in tropical dry forests: A case study from South Florida, USA. Ecological Applications, 15, 27-37.

Gould WA, Walker MD (1997) Landscape-scale patterns in plant species richness along an arctic river. Canadian Journal of Botany, 75, 1748-1765.

Guo QH, Liu J, Li YM, Zhai QP, Wang YC, Wu FF, Hu TY, Wan HW, Liu HM, Shen WM (2016) A near-surface remote sensing platform for biodiversity monitoring: Perspectives and prospects. Biodiversity Science, 24, 1249-1266. (in Chinese with English abstract) [郭庆华, 刘瑾, 李玉美, 翟 秋萍, 王永财, 吴芳芳, 胡天宇, 万华伟, 刘慧明, 申文 明 (2016) 生物多样性近地面遥感监测: 应用现状与前 景展望. 生物多样性, 24, 1249-1266.]

Guyon D, Guillot M, Vitasse Y, Cardot H, Hagolle O, Dezon S, Wigneron J (2011) Monitoring elevation variations in leaf phenology of deciduous broadleaf forests from SPOT/VEGETATION time-series. Remote Sensing of Environment, 115, 615-627.

Herrmann I, Pimstein A, Karnieli A (2011) LAI assessment of wheat and potato crops by VENIS and Sentinel-2 bands. Remote Sensing of Environment, 115, 2141-2151.

Hooper D, Chapin I, Ewel J, Hector A, Inchausti P, Lavorel S, Lawton J, Lodge D, Loreau M, Naeem S (2005) Effects of biodiversity on ecosystem functioning: A consensus of current knowledge. Ecological Monographs, 75, 3-35.

Hu HD, Li XY, Du YF, Zheng HF, Du BX, He XY (2012) Research advances in biodiversity remote sensing monitoring. Chinese Journal of Ecology, 31, 1591-1596. (in Chinese with English abstract) [胡海德, 李小玉, 杜宇飞, 郑 海峰, 都本绪, 何兴元 (2012) 生物多样性遥感监测方法 研究进展. 生态学杂志, 31, 1591-1596.]

Hu TY, Wang NN, Zhao XQ, Mi XC, Guo QH, Ma KP (2018) Advances in biodiversity observation network. Journal of Remote Sensing, 22, 708-711. (in Chinese with English abstract) [胡天宇, 王宁宁, 赵晓倩, 米湘成, 郭庆华, 马克 平 (2018) 生物多样性监测网络建设进展. 遥感学报, 22, 708-711.]

Jiang ZG, Ma KP (2009) Status, challenges and strategy in Conservation Biology. Biodiversity Science, 17, 107-116. (in Chinese with English abstract) [蒋志刚, 马克平 (2009) 保护生物学的现状、挑战和对策. 生物多样性, 17, 107-116.]

Jin R, Li X, Ma MG (2017) Key methods and experiment verification for the validation of quantitative remote sensing products. Advances in Earth Science, 32, 630-642. (in Chinese with English abstract) [晋锐, 李新, 马明国 (2017) 陆地定量遥感产品的真实性检验关键技术与试验验证. 地球科学进展, 32, 630-642.]

Keith DA, Rodríguez J, Rodríguez-Clark KM, Nicholson E,
Aapala K, Alonso Al, Asmussen M, Bachman S, Basset A, Barrow EG (2013) Scientific foundations for an IUCN Red List of Ecosystems. PLoS ONE, 8, e62111.

Li BV, Pimm S, Li S, Zhao L, Luo C (2017) Free-ranging livestock threaten the long-term survival of giant pandas. Biological Conservation, 216, 18-25.

Li X, Cheng G, Liu S (2013) Heihe watershed allied telemetry experimental research (HiWATER): Scientific objectives and experimental design. Bulletin of the American Meteorological Society, 94, 1145-1160.

Li X, Li X, Li Z (2009) Watershed allied telemetry experimental research. Journal of Geophysical Research: Atmospheres, 114.

Ma KP (2016) Hot topics for biodiversity science. Biodiversity Science, 24, 1-2. (in Chinese) [马克平 (2016) 生物多样性 科学的热点问题. 生物多样性, 24, 1-2.]

Ma KP, Qian YQ (1998) biodiversity conservation and its research progress. Chinese Journal of Applied and Environmental Biology, 4, 95-99. (in Chinese with English abstract) [马克平, 钱迎倩 (1998) 生物多样性保护及其研究进展. 应用与环境生物学报, 4, 95-99.]

Myers N, Mittermeier RA, Mittermeier CG, Fonseca D, Kent J (2000) Biodiversity hotspots for conservation priorities. Nature, 403, 853-858.

Nagendra H (2001) Using remote sensing to assess biodiversity. International Journal of Remote Sensing, 22, 2377-2400.

Oindo BO, Skidmore A (2002) Interannual variability of NDVI and species richness in Kenya. International Journal of Remote Sensing, 23, 285-298.

Palmer MW, Earls PG, Hoagland BW, White PS, Wohlgemuth $\mathrm{T}$ (2002) Quantitative tools for perfecting species lists. Environmetrics, 13, 121-137.

Petrou ZI, Kosmidou V, Manakos I, Stathaki T, Adamo M, Tarantino C (2014) A rule-based classification methodology to handle uncertainty in habitat mapping employing evidential reasoning and fuzzy logic. Pattern Recognition Letters, 48, 24-33.

Petrou ZI, Manakos I, Stathaki T (2015) Remote sensing for biodiversity monitoring: A review of methods for biodiversity indicator extraction and assessment of progress towards international targets. Biodiversity and Conservation, 24, 2333-2363.

Phillips SJ, Dudík M (2008) Modeling of species distributions with MaxEnt: New extensions and a comprehensive evaluation. Ecography, 31, 161-175.

Pimm SL, Jenkins CN, Abell R, Brooks TM, Gittleman JL, Joppa LN, Raven PH, Roberts CM, Sexton J (2014) The biodiversity of species and their rates of extinction, distribution, and protection. Science, 344, 1246752.

Pu R, Bell S, Levy KH, Meyer C (2010) Mapping detailed seagrass habitats using satellite imagery. IEEE Geoscience and Remote Sensing Symposium, Honolulu, USA, pp. 1-4.

Reiche M, Funk R, Zhang Z, Hoffmann C, Reiche J, Wehrhan 
M (2012) Application of satellite remote sensing for mapping wind erosion risk and dust emission-deposition in Inner Mongolia grassland, China. Grassland Science, 58, 8-19.

Rocchini D (2007) Effects of spatial and spectral resolution in estimating ecosystem $\alpha$-diversity by satellite imagery. Remote Sensing of Environment, 111, 423-434.

Rocchini D, Chiarucci A (2004) Testing the spectral variation hypothesis by using satellite multispectral images. Acta Oecologica, 26, 117-120.

Saatchi S, Buermann W, Mori S, Smith TB (2008) Modeling distribution of Amazonian tree species and diversity using remote sensing measurements. Remote Sensing of Environment, 112, 2000-2017.

Sellers P, Hall F, Margolis H (1995) The boreal ecosystem-atmosphere study (BOREAS): An overview and early results from the 1994 field year. Bulletin of the American Meteorological Society, 76, 1549-1577.

Sellers PJ, Hall FG, Kelly (1997) BOREAS in 1997: Experiment overview, scientific results, and future directions. Journal of Geophysical Research: Atmospheres, 102, 28731-28769.

Soberon J, Nakamura M (2009) Niches and distributional areas: Concepts, methods, and assumptions. Proceedings of the National Academy of Sciences, USA, 106, 19644-19650.

Tan J, Li A, Lei G, Bian J, Chen G, Ma K (2017) Preliminary assessment of ecosystem risk based on IUCN criteria in a hierarchy of spatial domains: A case study in Southwestern China. Biological Conservation, 215, 152-161.

Tan JB, Li AN, Lei GB, Chen GK, Ma KP (2017) Research advances and challenges in the IUCN Red List of Ecosystems. Biodiversity Science, 25, 453-463. (in Chinese with English abstract) [谭剑波 李爱农, 雷光斌, 陈国科, 马克 平 (2017) IUCN生态系统红色名录研究进展. 生物多样 性, 25, 453-463.]

Towsey M, Wimmer J, Williamson I, Roe P (2014) The use of acoustic indices to determine avian species richness in audio-recordings of the environment. Ecological Informatics, 21, 110-119.

Turner DP, Ritts WD, Cohen WB, Maeirsperger TK, Gower ST, Kirschbaum A, Running SW, Zhao M, Wofsy SC, Dunn AL, Law BE, Campbell JC, Oechel WC, Kwon HJ, Meyers TP, Small EE, Kurc SA, Gamon JA (2005) Site-level evaluation of satellite-based global terrestrial gross primary production and net primary production monitoring. Global Change Biology, 11, 666-684.

Turner W, Spector S, Gardiner N, Fladeland M, Sterling E, Steininger M (2003) Remote sensing for biodiversity science and conservation. Trends in Ecology \& Evolution, 18, 306-314.

Wang KY (2004) Processes of Subalpine Forest Ecosystems in the West of Sichuan. Sichuan Science and Technology Press, Chengdu. [王开运 (2004) 川西亚高山森林群落生 态系统过程. 四川科学技术出版社, 成都.]

Wang X, Wang Q, Wu C (2012) A method coupled with re- mote sensing data to evaluate non-point source pollution in the Xin'anjiang catchment of China. Science of the Total Environment, 430, 132-143.

Wei YC, Wu BF, Zhang XW, Du X (2008) Advances in remote sensing research for biodiversity monitoring. Advances in Earth Science, 23, 924-931. (in Chinese with English abstract) [魏彦昌, 吴炳方, 张喜旺, 杜金金 (2008) 生物多样 性遥感研究进展. 地球科学进展, 23, 924-931.]

Wulder MA, White JC, Coops NC, Butson CR (2008) Multi-temporal analysis of high spatial resolution imagery for disturbance monitoring. Remote Sensing of Environment, 112, 2729-2740.

Wythers KR, Reich PB, Turner DP (2003) Predicting leaf area index from scaling principles: Corroboration and consequences. Tree Physiology, 23, 1171-1179.

Xiao ZS, Li XH, Jiang GS (2014) Applications of camera trapping to wildlife surveys in China. Biodiversity Science, 22, 683-684. (in Chinese with English abstract) [肖治术, 李欣 海, 姜广顺 (2014) 红外相机技术在我国野生动物监测 研究中的应用. 生物多样性, 22, 683-684.]

Yin GF, Li AN, Verger A (2017) Spatiotemporally representative and cost-efficient sampling design for validation activities in Wanglang Experimental Site. Remote Sensing, 9, 1217.

Zainuddin M, Kiyofuji H, Saitoh K, Saitoh SI (2006) Using multi-sensor satellite remote sensing and catch data to detect ocean hot spots for albacore (Thunnus alalunga) in the northwestern North Pafíc. Deep -Sea Research II, 53, 419-431.

Zhang LB, Cui SP, Huang YJ, Chen DQ, Qiao HJ, Li CW, Jiang ZG (2014) Infrared camera traps in wildlife research and monitoring in China: Issues and insights. Biodiversity Science, 22, 696-703. (in Chinese with English abstract) [张 履冰, 崔绍朋, 黄元骏, 陈代强, 乔慧捷, 李春旺, 蒋志 刚 (2014) 红外相机技术在我国野生动物监测中的应用: 问题与限制. 生物多样性, 22, 696-703.]

Zhang Y, Liu QH, Tan LF, Huang HG, Ni WJ, Yin TG, Qin WH, Sun GQ (2017) A 3-D joint simulation platform for multiband remote sensing. IEEE Journal of Selected Topics in Applied Earth Observations and Remote Sensing, 10, 4763-4778.

Zhong L, Gong P, Biging GS (2014) ffifient corn and so ybean mapping with temporal extendability: A multi-year experiment using Landsat imagery. Remote Sensing of Environment, 140, 1-13.

Zhu C, Fang Y, Zhou KX, Mu SJ, Jiang JL (2015) IUCN red list of ecosystems: A new tool for biodiversity conservation. Acta Ecologica Sinica, 35, 2826-2836. (in Chinese with English abstract) [朱超, 方颖, 周可新, 穆少杰, 蒋金亮 (2015) 生态系统红色名录: 一种新的生物多样性保护工 具. 生态学报, 35, 2826-2836.] 